\title{
SUPERLUMINAL RADIO SOURCES
}

\author{
J. A. Zensus and T. J. Pearson \\ Owens Valley Radio Observatory \\ California Institute of Technology \\ Pasadena, California 91125 \\ USA
}

\begin{abstract}
There are now at least 23 radio sources in which apparent superluminal motion has been found. The effect is not limited to the "classic" examples like 3C 345, but is found in objects with a wide range of characteristic properties. We review the observations of the phenomenon and present an up-to-date list of the known superluminal sources.
\end{abstract}

\section{INTRODUCTION}

The discovery of superluminal sources in $1970-71$ is one of the most exciting results obtained in the twenty-year history of VLBI. It provided a remarkable confirmation of Martin Rees's prediction that "an object moving relativistically in suitable directions may appear to a distant observer to have a transverse velocity much greater than $c$ " (Rees 1966).

The development of the study of superluminal sources closely parallels that of VLBI. Rees's prediction of superluminal motion was made in response to the discovery of rapid variations in the radio flux of quasars (e.g., Dent 1965). Soon after the introduction of VLBI (Broten et al. 1967; Bare et al. 1967), variations in flux density were found to be accompanied by variations in source structure, which in some cases appeared to require faster-than-light expansion (e.g., Moffet et al. 1972). The first clear indication of superluminal expansion came when long-track observations of the quasars 3C 273 and 3C 279 revealed sharp visibility minima which moved in hour-angle between observing sessions (Knight et al. 1971; Whitney et al. 1971; Cohen et al. 1971). In the case of $3 \mathrm{C} 279$, the changes were most simply interpreted as due to an increase in the separation of a symmetric double source with a speed of about $10 c$.

Four such superluminal sources (3C 120, 3C 273, 3C 279, and 3C 345) were monitored in the period 1971-1977 (e.g., Cohen et al. 1977; Cotton et al. 1979); but some skepticism about the phenomenon persisted until real images of the sources were made using the structure information contained in the closure phase (Rogers et al. 1974; Readhead and Wilkinson 1978). For example, the sequence of images of $3 \mathrm{C} 273$ by Pearson et al. (1981) won a number of converts. Now, the basic phenomenon is no longer in doubt, and observers concentrate on unravelling the details of the trajectories and evolution of the components and on studying the statistics of superluminal sources, in the hope of determining the physical processes at work in the sources and of testing the predictions of models of extragalactic radio sources.

The Mark-III VLBI system has produced further discoveries. Its increased sen-

7

M. J. Reid and J. M. Moran (eds.), The Impact of VLBI on Astrophysics and Geophysics, 7-16.

(C) 1988 by the IAU. 
sitivity has allowed the detection of sources as weak as $50 \mathrm{mJy}$, and is essential for the study of superluminal motion in the weak cores of extended, lobe-dominated quasars. It has also permitted phase-referencing which can provide an absolute frame of reference for superluminal motion. VLBI imaging of polarization promises to be a very productive new technique. We confidently expect that the next technical steps forward, the Very Long Baseline Array and $\mathrm{mm}$ and space VLBI, will also provide new surprises and insights on the superluminal phenomenon.

Earlier reviews of observational results on superluminal sources include the articles by Cohen et al. (1977), Cohen and Unwin (1982, 1984), Kellermann and Pauliny-Toth (1981), Cohen (1986), and Porcas (1984, 1987). For a theoretical perspective we refer to, e.g., Scheuer (1984), Rees (1984), Phinney (1985), and Blandford (1984, 1987). Here we summarize recent observations and present a compilation of all known superluminal sources, without preempting the multitude of detailed results discussed at this Symposium. A more detailed account of the field can be found in the proceedings of the Workshop on Superluminal Radio Sources, held at Big Bear Solar Observatory in October 1986 (Zensus and Pearson 1987).

\section{CURRENT OBSERVATIONAL CAPABILITIES}

In spite of the great progress made in VLBI over the last 20 years, our understanding of superluminal sources is still limited by the instruments with which we study them.

VLBI images can now be made routinely at wavelengths $\lambda=50,18,6,2.8$, and $1.3 \mathrm{~cm}$, using as many as 18 antennas. The maximum resolution (FWHM) is $\sim 0.5 \lambda / D$; for baseline length $D=8200 \mathrm{~km}$ (Europe-California) and $\lambda=1.3 \mathrm{~cm}$ this is 0.2 milliarcseconds (mas). Most of the available images have been made at $6 \mathrm{~cm}$ and have resolution $\sim 1$ mas. Shorter wavelengths $(7 \mathrm{~mm}, 3 \mathrm{~mm})$ have been used successfully but are not yet routine, and image-forming capability is crude.

In terms of linear resolution, 1 mas ranges from about $0.25 \mathrm{pc}$ in nearby active galactic nuclei to about $4 \mathrm{pc}$ in most quasars. For comparison, the Schwarzschild radius of a $10^{9} M_{\odot}$ black hole is $\sim 10^{-4} \mathrm{pc}$, the size of the "broad emission line region" in active nuclei is typically $\sim 1 \mathrm{pc}$, and the size of the "narrow emission line region" is typically $\sim 100$ pc. Thus VLBI can resolve the broad-line region, but it is still some way from resolving the "central engine".

When making VLBI images, self-calibration techniques are used to circumvent atmospheric and oscillator phase and amplitude instability (e.g., Pearson and Readhead $1984 a$ ). These techniques require a source to be detectable in the coherence time (in the range $30 \mathrm{~s}$ to $10 \mathrm{~min}$, depending on frequency and weather conditions), so that observations are restricted to strong sources, brighter than $\sim 0.5 \mathrm{Jy}$ for the Mark II recording system (bandwidth $2 \mathrm{MHz}$ ), or $\sim 0.1 \mathrm{Jy}$ for the Mark III system (bandwidth up to $112 \mathrm{MHz}$ ). When self-calibration is used, absolute position information is lost, which makes it difficult to register images made at different wavelengths or epochs.

The dynamic range achieved in VLBI images (the ratio of the strongest feature in the image to the noise level) ranges from about $20: 1$ to $1000: 1$, and is limited by (a) sensitivity (a few millijanskys per beam area), (b) "non-closing" calibration errors which cannot be removed by self-calibration, and $(c)$ inadequate coverage of the $(u, v)$ plane (i.e., missing Fourier components of the image). All of these problems should be alleviated when the Very Long Baseline Array and other new arrays are completed in the 1990s. 


\section{APPARENT VELOCITY}

What is measured in superluminal sources is the rate of change of the apparent separation of two features in the brightness distribution. To convert this angular velocity $\mu$ into an apparent linear velocity $v_{\mathrm{app}}=c \beta_{\mathrm{app}}$ (projected on the sky), the distance to the source is needed, and for almost all the known superluminal sources this must be inferred from the redshift, $z$. In the standard Friedmann-Robertson-Walker model, parameterized by the Hubble constant $H_{0}$ and the deceleration parameter $q_{0}$, the apparent transverse velocity $\beta_{\text {app }}$ is related to the observed angular velocity $\mu$ by

$$
\beta_{\mathrm{app}}=\frac{\mu z}{H_{0}(1+z)}\left(\frac{1+\sqrt{1+2 q_{0} z}+z}{1+\sqrt{1+2 q_{0} z}+q_{0} z}\right) .
$$

The values of $H_{0}$ and $q_{0}$ are unfortunately still subject to debate. For consistency, we shall use $H_{0}=100 \mathrm{hm} \mathrm{s}^{-1} \mathrm{Mpc}^{-1}$ and $q_{0}=0.5$ in this review. If $\mu$ is measured in milliarcseconds per year, the appropriate numerical factor in equation (1) is $H_{0}=2.11 \times$ $10^{-2} h$ mas $\mathrm{yr}^{-1}$.

\section{3C 345: A SUPERLUMINAL ARCHETYPE}

The quasar 3C 345 is in many respects the best-studied superluminal source owing to its high radio flux density and its high declination $\left(39^{\circ}\right)$ which allow routine Mark-II VLBI monitoring with many stations and good $(u, v)$-coverage. Figure 1 shows a sequence of hybrid maps of this source made at $10.7 \mathrm{GHz}$ (Biretta, Moore, and Cohen 1986).

The compact structure of $3 \mathrm{C} 345$ has the typical asymmetric "core-jet" character, with several "jet" components separating superluminally from the "core" $D$. The identification of core and jet components in superluminal sources is usually based on the finding that one component, the core, is compact, located at one end of the structure, and has a flat radio spectrum, whereas the jet components tend to be resolved and have steeper spectra. All too often, however, such a distinction is based on an educated guess rather than firm observational evidence. A related problem is the difficulty of uniquely identifying components from one epoch to another (e.g., Blandford 1987). In 3C 345, the phase-referencing observations of Bartel et al. (1986) have shown that the core indeed appears stationary on the sky and that any motion with respect to the nearby quasar NRAO 512 is smaller than 0.2 mas per year. At least three superluminal components $(\mathrm{C} 2, \mathrm{C} 3, \mathrm{C} 4)$ in 3C 345 have now been monitored for several years. Their properties are compared with the predictions of a synchrotron self-Compton model by Biretta and Cohen at this Symposium. Here we list some of the observational results.

1. Component sizes and separation from the core can be frequency-dependent, as seen for C3 in 3C 345 .

2. Superluminal components in a given source do not always all move with the same velocity. This is seen not only in $3 \mathrm{C} 345$, but also in $3 \mathrm{C} 120$ and $3 \mathrm{C} 279$, and several other sources.

3. Acceleration is seen for component $\mathrm{C} 4$ in $3 \mathrm{C} 345$ with changes in both magnitude and direction of the superluminal velocity.

4. The size of the superluminal components in $3 \mathrm{C} 345$ increases roughly linearly with separation from the core, corresponding to a projected jet opening angle of $26^{\circ}$.

5. An important question is whether individual components follow the same path or are moving in different directions. There is some evidence that components in 3C 345 (and also in 3 C 273) follow a common curved path, but this is still tentative. 


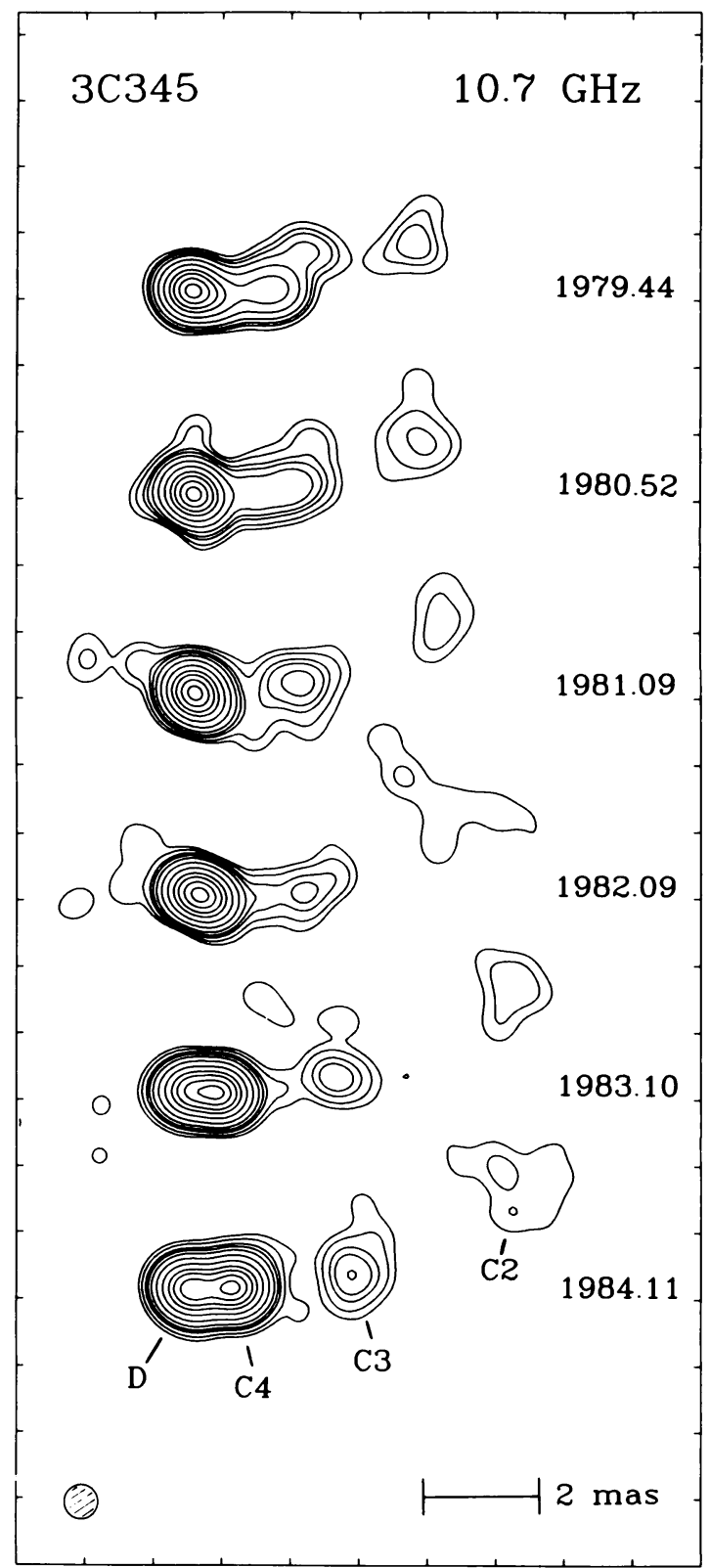

Figure 1 A sequence of hybrid maps of $3 \mathrm{C} 345$ at $10.7 \mathrm{GHz}$ (from Biretta, Moore, and Cohen 1986). North is at the top; tick marks are 1.2 mas apart. Contour levels are $0.5,1,2,3,5,10,20,35,50,70$, and $90 \%$ of the peak intensity; lower contours have been omitted in some of the maps. The FWHM of the Gaussian restoring beam is 0.6 mas (hatched circle). 
6. The structure in 3C 345 is curved northward in the direction of the extended emission regions located 1-3 arcsec from the core. Such curvature is quite common. The early finding that curvature and also misalignments with the large-scale symmetry axis are more pronounced in core-dominated sources has been confirmed.

Although some of the above properties have been seen only in $3 \mathrm{C} 345$ it is likely that this object is typical of the class of superluminal sources that contain strong, core-dominated sources as found in radio samples selected at high frequencies.

\section{SEARCHING FOR SUPERLUMINAL SOURCES}

The first few superluminal sources were discovered accidentally in observations of the brightest compact sources in the sky. These include 3C 345, 3C 273, 3C 279, and 3C 120. In one case, NRAO 140, superluminal motion was successfully predicted based on its low X-ray intensity. Marscher (1987) has recently cautioned that such predictions may be much weaker than previously thought, owing to ill-defined parameters in the assumed synchrotron self-Compton models.

Recently, a number of systematic searches for superluminal sources have been undertaken. Such programs are essential for determining the frequency of occurrence of superluminal motion and the distribution of apparent velocities. Witzel and his colleagues (Witzel 1987; Eckart et al. 1986, 1987) have selected a sample of 13 sources from the NRAO-MPIfR 5-GHz S5 survey, with flux densities $\geq 1 \mathrm{Jy}$ and flat spectra. They have studied these sources intensively in the $\mathrm{X}$-ray, optical, and radio wavebands, including multi-epoch VLBI at several frequencies. Three of the sources with known redshift show superluminal motion $(0212+735,1150+812,1928+738)$ and two more are superluminal candidates. Four sources without redshifts show apparent expansion which would be superluminal if, as seems probable, the redshifts exceed 0.3 . There is also evidence from a comparison of measured and predicted inverse-Compton $\mathrm{X}$-ray fluxes for bulk relativistic motion in 12 of the 13 sources. They conclude that bulk relativistic motion is a common phenomenon in core-dominated sources.

Pearson, Readhead, and Barthel (1987; see also Pearson and Readhead 1984b) are studying a larger sample of 65 sources, also selected at $5 \mathrm{GHz}$; the selection criterion was flux density $\geq 1.3 \mathrm{Jy}$, with no spectral selection. Forty-five of these sources are strong enough to map with Mark-II VLBI. Although the observations are not yet complete, nine sources in this sample are already known to be superluminal; four of these are new $(0850+581,0906+430,1642+690$, and $1928+738)$. Superluminal sources are found in all types of source except the "compact double" sources (Phillips and Mutel 1982; Hodges and Mutel 1987).

Both of the above programs have selected strong sources. If the beaming theories are correct, sources in such flux-density limited samples are not randomly oriented but are preferentially beamed towards the observer. To avoid this bias, several groups have attempted to define orientation-unbiased samples for study, selecting sources by the flux density of the extended lobes, which are assumed to be unbeamed, rather than the compact core. Hough and Readhead (1987) are studying an unbiased sample of 26 double-lobed quasars using the Mark-III system at $10.7 \mathrm{GHz}$. So far they have studied six sources and found two of them to be superluminal (3C 245 and 3C 263). Zensus and Porcas (1987) have selected a similar sample of 30 quasars, based on the flux density at $966 \mathrm{MHz}$ where the core is not a major contributor. Of the five sources they have studied closely, three are superluminal (3C 179, 3C 263, and 1951+498). The results from both studies indicate a trend that velocities in lobe-dominated superluminals are smaller than in core-dominated objects, in agreement with the expectations of beaming models. 
Table 1 Superluminal Radio Sources

\begin{tabular}{|c|c|c|c|c|c|c|}
\hline Source & & $z$ & Component & $\mu\left[\operatorname{mas} \mathrm{yr}^{-1}\right]$ & $\beta_{\text {app }} h$ & Ref. \\
\hline $0212+735$ & & 2.367 & - & $0.09^{a}$ & 3.9 & 1 \\
\hline $0333+321$ & NRAO 140 & 1.258 & B & 0.15 & 4.8 & 2 \\
\hline \multirow[t]{5}{*}{$0430+052$} & $3 C 120$ & 0.033 & $\mathbf{A}$ & 1.35 & 2.1 & 3 \\
\hline & & & B & 2.53 & 3.9 & 3 \\
\hline & & & $\mathbf{C}$ & 2.47 & 3.8 & 3 \\
\hline & & & D & 2.66 & 4.1 & 3 \\
\hline & & & $\mathbf{E}$ & 2.54 & 3.9 & 3 \\
\hline $0723+679$ & $3 C 179$ & 0.846 & - & 0.19 & 4.8 & 4 \\
\hline $0735+178$ & & 0.424 & NE & 0.18 & 2.8 & 5 \\
\hline $0850+581$ & & 1.322 & - & 0.12 & 3.9 & 6 \\
\hline $0851+202$ & OJ 287 & 0.306 & SW1-2 & $0.28^{b}$ & 3.3 & 7 \\
\hline $0906+430$ & $3 C 216$ & 0.669 & - & 0.11 & 2.4 & 8 \\
\hline \multirow[t]{2}{*}{$0923+392$} & $4 C 39.25$ & 0.699 & $a-c$ & $<0.006^{c}$ & $<0.1$ & 9 \\
\hline & & & $\mathbf{b}$ & 0.16 & 3.5 & 9 \\
\hline $1040+123$ & $3 C 245$ & 1.029 & - & $0.11^{d}$ & 3.1 & 10 \\
\hline $1137+660$ & $3 C 263$ & 0.652 & - & 0.06 & 1.3 & 11 \\
\hline $1150+812$ & & 1.25 & - & 0.13 & 4.1 & 1 \\
\hline \multirow[t]{4}{*}{$1226+023$} & $3 C 273$ & 0.158 & C3 & 0.79 & 5.3 & 12 \\
\hline & & & C4 & 0.99 & 6.6 & 12 \\
\hline & & & C5 & 1.20 & 8.1 & 13 \\
\hline & & & C7a & 0.76 & 5.1 & 13 \\
\hline \multirow[t]{2}{*}{$1253-055$} & $3 C 279$ & 0.538 & $?$ & $0.5^{e}$ & 9.2 & 14 \\
\hline & & & B2 & 0.11 & 2.0 & 15 \\
\hline \multirow[t]{3}{*}{$1641+399$} & $3 C 345$ & 0.595 & $\mathrm{C} 2$ & 0.48 & 9.5 & 16 \\
\hline & & & C3 & 0.30 & 5.9 & 16 \\
\hline & & & C4 & $0.07,0.3$ & $1.4,5.9$ & 16 \\
\hline $1642+690$ & & 0.751 & - & 0.34 & 7.9 & 17 \\
\hline $1721+343$ & $4 \mathrm{C} 34.47$ & 0.206 & - & 0.36 & 3.1 & 18 \\
\hline \multirow[t]{2}{*}{$1901+319$} & 3C 395 & 0.635 & 1-2 & $<0.06^{c}$ & $<1.2$ & 19 \\
\hline & & & 3 & $0.64^{d}$ & 13.2 & 19 \\
\hline $1928+738$ & & 0.302 & A1-4 & 0.6 & 7.0 & 20 \\
\hline $1951+498$ & & 0.466 & - & $\sim 0.07^{d}$ & $\sim 1.2$ & 21 \\
\hline $2200+420$ & BL Lac & 0.0695 & $1-4$ & $\sim 0.76^{f}$ & $\sim 2.4$ & 22 \\
\hline $2230+114$ & CTA 102 & 1.037 & - & $\sim 0.65^{g}$ & $\sim 18.5$ & 23 \\
\hline \multirow[t]{2}{*}{$2251+158$} & $3 \mathrm{C} 454.3$ & 0.859 & 2 & $<0.05$ & $<1.3$ & 24 \\
\hline & & & 4 & $0.35^{h}$ & 8.9 & 24 \\
\hline
\end{tabular}

Among other programs to search for superluminal sources, Bååth (1987) has studied several low-frequency variable sources, and found one superluminal (CTA $102=$ $2230+114)$. This source has an unusually high expansion speed, $(18 \pm 4) h^{-1} c$, which may be related to the high Lorentz factors required to explain low-frequency variability as an intrinsic effect. The discovery of superluminal motion lends some support to the idea that the variability is indeed intrinsic rather than due to refractive scintillation in the interstellar medium. Båath (1984) has also studied a number of BL-Lac objects and has found one of these to be superluminal $(0735+178)$.

\section{INVENTORY}

At the last IAU meeting on VLBI (IAU Symposium No. 110 in Bologna), eight sources were known to show apparent superluminal motion (Cohen and Unwin 1984), but since then the number has increased rapidly and is now at least 23 . In Table 1 we have assembled a list of all superiuminal sources known at the time of writing (May 1987), including redshift, apparent angular velocity, and inferred apparent velocity. The list includes the "classic" sources, the sources discovered in the surveys described above, and a few more individual 
Table 2 Subluminal and Stationary Sources

\begin{tabular}{lllcccc}
\hline \multicolumn{1}{c}{ Source } & & $z$ & Component & $\mu\left[\operatorname{mas~yr}^{-1}\right]$ & $\beta_{\text {app }} h$ & Ref. \\
\hline $0153+744$ & & 2.33 & - & $<0.03^{i}$ & $<1.3$ & 1 \\
$0316+413$ & 3C 84 & 0.0172 & - & 0.24 & 0.2 & 25 \\
$0710+439$ & & 0.517 & B & $<0.04^{j}$ & $<0.7$ & 8 \\
& & C & $<0.07^{j}$ & $<1.3$ & 8 \\
$0711+356$ & & - & -0.05 & -1.8 & 26 \\
$1228+127$ & M 87 & 0.004 & - & $<0.3$ & $<0.1$ & 27 \\
$1637+826$ & NGC 6251 & 0.023 & - & $<0.3$ & $<0.3$ & 28 \\
$1934-638$ & & 0.183 & - & $<0.4^{k}$ & $<3.1$ & 29 \\
$2021+614$ & & 0.2266 & - & $<0.04$ & $<0.4$ & 26 \\
$2134+004$ & & 1.936 & $<0.01^{h}$ & $<0.4$ & 30 \\
\hline \hline
\end{tabular}

Notes to Tables 1 and 2: ${ }^{a}$ no published sequence of maps available yet. $\quad{ }^{b}$ polarization data. $\quad c$ limit on velocity between stationary components. ${ }^{d}$ well-separated double structure only at 2 epochs. $e$ onebaseline results. $\quad f$ average of four events; possibly deceleration. $g$ maps made at $932 \mathrm{MHz}$; wellseparated components only at 2 epochs. ${ }^{h}$ complex structure. ${ }^{i}$ upper limit on separation of 12-mas double structure. $j$ 1- $\sigma$ limit; motion relative to component A. $k$ 12-yr limit.

References in Tables 1 and 2: 1. Witzel 1987. 2. Marscher and Broderick 1985. 3. Walker, Benson, and Unwin 1987. 4. Porcas 1987. 5. Bååth 1984. 6. Barthel et al. 1986. 7. Roberts and Wardle 1987. 8. Pearson, Readhead, and Barthel 1987. 9. Shaffer et al. 1987. 10. Hough and Readhead 1987. 11. Zensus, Hough, and Porcas 1987. 12. Unwin et al. 1985. 13. Cohen et al. 1987. 14. Cotton et al. 1979. 15. Unwin 1987. 16. Biretta, Moore, and Cohen 1986. 17. Pearson et al. 1986. 18. Barthel 1987. 19. Simon et al. 1987. 20. Eckart et al. 1985. 21. Zensus and Porcas 1987. 22. Mutel and Phillips 1987. 23. Bååth 1987. 24. Pauliny-Toth 1987. 25. Romney et al. 1984. 26. Readhead, Pearson, and Unwin 1984. 27. Schmitt and Reid 1985. 28. Jones 1986. 29. A. K. Tzioumis, private communication. 30. Pauliny-Toth et al. 1984.

objects. We have tried to restrict the list to sources mapped at several epochs, where component motion can be clearly followed from one epoch to the next, and an accurate proper motion can be measured. Few authors are brave enough to attach error estimates to their measurements, so we have not included any in the table.

In Table 2, we have listed a miscellaneous collection of additional sources. These include a few which have been studied well enough to establish definite upper limits on any motion. Some of the upper limits are subluminal; but only two sources, NGC 1275 and $\mathrm{M} 87$, have measured non-zero subluminal velocities. Comments on these sources are included in the notes below the table. There are also several sources which have shown "superluminal behavior" but for which we think it is premature to estimate a velocity $(3 \mathrm{C} 446,0235+164,3 \mathrm{C} 147,3 \mathrm{C} \mathrm{371})$.

\section{SOME INTERESTING SOURCES}

The galaxy 3C 120 is the closest superluminal source and the detailed observations by Walker, Benson, and Unwin (1987) have revealed a jet with continuous properties on scales from $0.5 h^{-1} \mathrm{pc}$ to more than $100 h^{-1} \mathrm{kpc}$, indicating that the jet characteristics are defined in the regions of the superluminal components.

The quasar 3C 179 stands out from the small group of established superluminal sources. This source is the first classical double radio source with a relatively weak core in which superluminal motion was found. Although much weaker, the components in this source exhibit properties similar to those in 3C 345 (Porcas 1987). It seems likely that 3C 179 is typical of the weak cores that are now being studied in well-defined samples.

The quasar 4C 39.25 was earlier considered the standard example of a source that does not show superluminal components, but rather has a stationary double structure. Subsequent observations indicated an apparent superluminal contraction, but it has now 
become clear that we see a superluminal component moving between two stationary components (Shaffer et al. 1987). It is interesting that none of these components appears to have the typical properties of a core. A similar situation is present in the quasar 3C 395, but new results shown at this Symposium by Simon et al. indicate that the superluminal motion has stopped. Deceleration of components to rest is also seen in BL Lac (Mutel and Phillips 1987). There is still no clear evidence in any source for contractions, although the identification of changes as expansion is not in all cases unique.

The quasar 3C 454.3 appears to be a good example of an object that exhibits rapid brightness changes in a stationary structure, in this case together with superluminally moving components (Pauliny-Toth 1987).

\section{CONCLUSIONS}

Superluminal motion is now established as a common phenomenon in active galactic nuclei. The available observational results are beginning to constrain the parameters of physical models for individual sources, and to test more general source models (e.g., Cohen et al., this Symposium). While relativistic beaming clearly does play a role in compact radio sources (e.g., Blandford 1987), difficulties such as as the problem of exceedingly large linear sizes of superluminal sources (e.g., Schilizzi and de Bruyn 1983; Browne 1987; Barthel et $a l$., this Symposium) seem to require modifications of the most naïve beaming theories. Future work will increasingly focus on the comparison of superluminal sources with other classes of object in the quest for unified models of extragalactic sources. For example, a connection between superluminal sources and blazars is being discussed (e.g., Impey 1987; Rudnick 1987).

\section{REFERENCES}

Bååth, L. B. 1984, in IAU Symposium 110, VLBI and Compact Radio Sources, ed. R. Fanti, K. Kellermann, and G. Setti (Dordrecht: Reidel), p. 127.

Bååth, L. B. 1987, in Superluminal Radio Sources, ed. J. A. Zensus and T. J. Pearson (Cambridge: Cambridge University Press), p. 206.

Bare, C., Clark, B. G., Kellermann, K. I., Cohen, M. H., and Jauncey, D. L. 1967, Science, $157,189$.

Bartel, N., Herring, T. A., Ratner, M. I., Shapiro, I. I., and Corey, B. E. 1986, Nature, $319,733$.

Barthel, P. D. 1987, in Superluminal Radio Sources, ed. J. A. Zensus and T. J. Pearson (Cambridge: Cambridge University Press), p. 148.

Barthel, P. D., Pearson, T. J., Readhead, A. C. S., and Canzian, B. J. 1986, Astrophys. J. (Letters), 310 , L7.

Biretta, J. A., Moore, R. L., and Cohen, M. H. 1986, Astrophys. J., 308, 93.

Blandford, R. D. 1984, in IAU Symposium 110, VLBI and Compact Radio Sources, ed. R. Fanti, K. Kellermann, and G. Setti (Dordrecht: Reidel), p. 215.

Blandford, R. D. 1987, in Superluminal Radio Sources, ed. J. A. Zensus and T. J. Pearson (Cambridge: Cambridge University Press), p. 310.

Broten, N. W., Legg, T. H., Locke, J. L., McLeish, C. W., Richards, R. S., Chisholm, R. M., Gush, H. P., Yen, J. L., and Galt, J. A. 1967, Nature, 215, 38.

Browne, I. W. A. 1987, in Superluminal Radio Sources, ed. J. A. Zensus and T. J. Pearson (Cambridge: Cambridge University Press), p. 129.

Cohen, M. H. 1986, in Highlights of Modern Astrophysics, ed. S. L. Shapiro and S. A. Teukolsky (New York: Wiley), p. 299.

Cohen, M. H., Cannon, W., Purcell, G. H., Shaffer, D. B., Broderick, J. J., Kellermann, K. I., and Jauncey, D. L. 1971, Astrophys. J., 170, 207.

Cohen, M. H., Kellermann, K. I., Shaffer, D. B., Linfield, R. P., Moffet, A. T., Romney, J. D., Seielstad, G. A., Pauliny-Toth, I. I. K., Preuss, E., Witzel, A., Schilizzi, R. T., and Geldzahler, B. J. 1977, Nature, 268, 405. 
Cohen, M. H., and Unwin, S. C. 1982, in IAU Symposium 110, VLBI and Compact Radio Sources, ed. R. Fanti, K. Kellermann, and G. Setti (Dordrecht: Reidel), p. 95.

Cohen, M. H., and Unwin, S. C. 1984, in IAU Symposium 97, Extragalactic Radio Sources, ed. D. S. Heeschen and C. M. Wade (Dordrecht: Reidel), p. 345.

Cohen, M. H., Zensus, J. A., Biretta, J. A., Comoretto, G., Kaufmann, P., and Abraham, Z. 1987, Astrophys. J. (Letters), 315, L89.

Cotton, W. D., Counselman III, C. C., Geller, R. B., Shapiro, I. I., Wittels, J. J., Hinteregger, H. F., Knight, C. A., Rogers, A. E. E., Whitney, A. R., and Clark, T. A. 1979, Astrophys. J. (Letters), 229, L115.

Dent, W. A. 1965, Science, 148, 1458.

Eckart, A., Witzel, A., Biermann, P., Johnston, K. J., Simon, R., Schalinski, C., and Kühr, H. 1986, Astron. Astrophys., 168, 17.

Eckart, A., Witzel, A., Biermann, P., Johnston, K. J., Simon, R., Schalinski, C., and Kühr, H. 1987, Astron. Astrophys. Suppl., 67, 121.

Eckart, A., Witzel, A., Biermann, P., Pearson, T. J., Readhead, A. C. S., and Johnston, K. J. 1985, Astrophys. J. (Letters), 296, L23.

Hodges, M. W., and Mutel, R. L. 1987, in Superluminal Radio Sources, ed. J. A. Zensus and T. J. Pearson (Cambridge: Cambridge University Press), p. 168.

Hough, D. H., and Readhead, A. C. S. 1987, in Superluminal Radio Sources, ed. J. A. Zensus and T. J. Pearson (Cambridge: Cambridge University Press), p. 114.

Impey, C. 1987, in Superluminal Radio Sources, ed. J. A. Zensus and T. J. Pearson (Cambridge: Cambridge University Press), p. 233.

Jones, D. L. 1986, Astrophys. J. (Letters), 309, L5.

Kellermann, K. I., and Pauliny-Toth, I. I. K. 1981, Ann. Rev. Astron. Astrophys., $19,373$.

Knight, C. A., Robertson, D. S., Rogers, A. E. E., Shapiro, I. I., Whitney, A. R., Clark, T. A., Goldstein, R. M., Marandino, G. E., and Vandenberg, N. R. 1971, Science, 172, 52.

Marscher, A. P. 1987, in Superluminal Radio Sources, ed. J. A. Zensus and T. J. Pearson (Cambridge: Cambridge University Press), p. 280.

Marscher, A. P., and Broderick, J. J. 1985, Astrophys. J., 290, 735.

Moffet, A. T., Gubbay, J., Robertson, D. S., and Legg, A. J. 1972, in IAU Symposium 44, External Galaxies and Quasi-Stellar Objects, ed. D. S. Evans (Dordrecht: Reidel), p. 228.

Mutel, R. L., and Phillips, R. B. 1987, in Superluminal Radio Sources, ed. J. A. Zensus and T. J. Pearson (Cambridge: Cambridge University Press), p. 60.

Pauliny-Toth, I. I. K. 1987, in Superluminal Radio Sources, ed. J. A. Zensus and T. J. Pearson (Cambridge: Cambridge University Press), p. 55.

Pauliny-Toth, I. I. K., Porcas, R. W., Zensus, A., and Kellermann, K. I. 1984, in IAU Symposium 110, VLBI and Compact Radio Sources, ed. R. Fanti, K. Kellermann, and G. Setti (Dordrecht: Reidel), p. 149.

Pearson, T. J., Barthel, P. D., Lawrence, C. R., and Readhead, A. C. S. 1986, Astrophys. J. (Letters), $300, \mathrm{~L} 25$.

Pearson, T. J., and Readhead, A. C. S. 1984a, Ann. Rev. Astron. Astrophys., $22,97$.

Pearson, T. J., and Readhead, A. C. S. 1984b, in IAU Symposium 110, VLBI and Compact Radio Sources, ed. R. Fanti, K. Kellermann, and G. Setti (Dordrecht: Reidel), p. 15.

Pearson, T. J., Readhead, A. C. S., and Barthel, P. D. 1987, in Superluminal Radio Sources, ed. J. A. Zensus and T. J. Pearson (Cambridge: Cambridge University Press), p. 94.

Pearson, T. J., Unwin, S. C., Cohen, M. H., Linfield, R. P., Readhead, A. C. S., Seielstad, G. A., Simon, R. S., and Walker, R. C. 1981, Nature, 290, 365.

Phillips, R. B., and Mutel, R. L. 1982, Astron. Astrophys., 106, 21.

Phinney, E. S. 1985, in Astrophysics of Active Galaxies and Quasi-Stellar Objects, ed. J. S. Miller (Mill Valley: University Science Books), p. 453.

Porcas, R. W. 1984, in Active Galactic Nuclei, ed. J. E. Dyson (Manchester: Manchester University Press), p. 20.

Porcas, R. W. 1987, in Superluminal Radio Sources, ed. J. A. Zensus and T. J. Pearson (Cambridge: Cambridge University Press), p. 12.

Readhead, A. C. S., Pearson, T. J., and Unwin, S. C. 1984, in IAU Symposium 110, VLBI and Compact Radio Sources, ed. R. Fanti, K. Kellermann, and G. Setti (Dordrecht: Reidel), p. 131.

Readhead, A. C. S., and Wilkinson, P. N. 1978, Astrophys. J., 223, 25.

Rees, M. J. 1966, Nature, 211, 468.

Rees, M. J. 1984, in IAU Symposium 110, VLBI and Compact Radio Sources, ed. R. Fanti, K. Kellermann, and G. Setti (Dordrecht: Reidel), p. 207.

Roberts, D. H., and Wardle, J. F. C. 1987, in Superluminal Radio Sources, ed. J. A. Zensus and T. J. Pearson (Cambridge: Cambridge University Press), p. 193. 
Rogers, A. E. E., Hinteregger, H. F., Whitney, A. R., Counselman, C. C., Shapiro, I. I., Wittels, J. J., Klemperer, W. K., Warnock, W. W., Clark, T. A., Hutton, L. K., Marandino, G. E., Rönnäng, B. O., and Rydbeck, O. E. H. 1974, Astrophys. J., 193, 293.

Romney, J. D., Alef, W., Pauliny-Toth, I. I. K., Preuss, E., and Kellermann, K. I. 1984, in IAU Symposium 110, VLBI and Compact Radio Sources, ed. R. Fanti, K. Kellermann, and G. Setti (Dordrecht: Reidel), p. 137.

Rudnick, L. 1987, in Superluminal Radio Sources, ed. J. A. Zensus and T. J. Pearson (Cambridge: Cambridge University Press), p. 217.

Scheuer, P. A. G. 1984, in IAU Symposium 110, VLBI and Compact Radio Sources, ed. R. Fanti, K. Kellermann, and G. Setti (Dordrecht: Reidel), p. 197.

Schilizzi, R. T., and de Bruyn, A. G. 1983, Nature, 303, 26.

Schmitt, J. H. M. M., and Reid, J. M. 1985, Astrophys. J., 289, 120.

Shaffer, D. B., Marscher, A. P., Marcaide, J., and Romney, J. D. 1987, Astrophys. J. (Letters), 314, L1.

Simon, R. S., Johnston, K. J., Hall, J., Spencer, J. H., and Waak, J. A. 1987, in Superluminal Radio Sources, ed. J. A. Zensus and T. J. Pearson (Cambridge: Cambridge University Press), p. 72.

Unwin, S. C. 1987, in Superluminal Radio Sources, ed. J. A. Zensus and T. J. Pearson (Cambridge: Cambridge University Press), p. 34.

Unwin, S. C., Cohen, M. H., Biretta, J. A., Pearson, T. J., Seielstad, G. A., Walker, R. C., Simon, R. S., and Linfield, R. P. 1985, Astrophys. J., 289, 109.

Walker, R. C., Benson, J. M., and Unwin, S. C. 1987, in Superluminal Radio Sources, ed. J. A. Zensus and T. J. Pearson (Cambridge: Cambridge University Press), p. 48.

Whitney, A. R., Shapiro, I. I., Rogers, A. E. E., Robertson, D. S., Knight, C. A., Clark, T. A., Goldstein, R. M., Marandino, G. E., and Vandenberg, N. R. 1971, Science, 173, 225.

Witzel, A. 1987, in Superluminal Radio Sources, ed. J. A. Zensus and T. J. Pearson (Cambridge: Cambridge University Press), p. 83.

Zensus, J. A., Hough, D. H., and Porcas, R. W. 1987, Nature, 325, 36.

Zensus, J. A., and Pearson, T. J. (ed.) 1987, Superluminal Radio Sources (Cambridge: Cambridge University Press).

Zensus, J. A., and Porcas, R. W. 1987, in Superluminal Radio Sources, ed. J. A. Zensus and T. J. Pearson (Cambridge: Cambridge University Press), p. 126. 